\title{
STRUCTURAL IMAGING MARKERS FOR THERAPEUTIC TRIALS IN ALZHEIMER'S DISEASE
}

\author{
N.C. FOX, J. KENNEDY \\ Dementia Research Centre, Department of Neurodegenerative Disease, UCL Institute of Neurology, Queen Square, London WC1N 3BG, UK
}

\section{Introduction}

Structural imaging has long had a role in entry criteria for $\mathrm{AD}$ trials - in current practice this means magnetic resonance imaging (MRI). The advent of potential disease-modifying therapies has led to interest in using MRI as an outcome measure - for safety and, importantly, to assess effects on disease progression. This article addresses the roles for structural imaging within trials of treatments for $\mathrm{AD}$ and mild cognitive impairment (MCI) and focuses on the use of MRI as an outcome.

\section{Selecting and defining the trial population: exclusion and inclusion criteria}

\section{Exclusion}

Structural imaging has been part of the entry criteria for trials of treatments for $\mathrm{AD}$ for almost as long as such trials have been conducted (1). Initially computed tomography (CT) or MRI was used to exclude conditions such as a brain tumour or a haematoma. These are relatively rare and much more relevant is to exclude vascular causes of dementia which may mimic AD. In particular, patients with small-vessel vascular dementia may fulfil clinical criteria for entry into AD trials. The most practical way to identify patients with a vascular cause of their cognitive impairment is by the use of MRI. This has the advantage of a more homogeneous trial population with a higher proportion of subjects with $\mathrm{AD}$ as their primary pathology. The disadvantages are cost, delays in recruitment and the argument that the trial population is no longer representative of the typical patient where vascular pathology often co-exists with Alzheimer's disease. The balance between excluding too few or too many subjects may be a difficult one to achieve. Decisions on exclusion can most reliably be achieved through central reading with standard MRI protocols, but the exact cut-offs between what is an acceptable or unacceptable vascular load remain arbitrary. A concomitant advantage of quantifying baseline vascular load in the included patients is that it may be defined in the statistical analysis plan as a covariate for both clinical and imaging outcomes.

\section{Inclusion}

Medial temporal lobe atrophy is an early and defining feature of $\mathrm{AD}$ and is predictive of progression from mild cognitive impairment (MCI) to $\mathrm{AD}(2,3)$. This recognition has led to MRI being incorporated in trial inclusion criteria. The aim is to include those individuals more likely to have AD.
When applied to MCI studies this has a potential to enrich recruitment of subjects with a higher likelihood of progression during the study. Candidate medial temporal lobe structures include hippocampus, entorhinal cortex and the amygdala (4). The hippocampus has by far and away been the most studied. Hippocampal or medial temporal lobe atrophy is usually assessed visually (e.g. Scheltens rating scale) for inclusion criteria (1).

Moving towards disease prevention studies, there is interest in using rates of change either of whole brain or hippocampus to identify individuals even earlier: those who are at high risk of $\mathrm{AD}$ who may already have started to have accelerating atrophy as they progress to MCI or AD (5). At-risk individuals might be those with a family history, a known genetic risk or those identified through CSF biomarkers or amyloid imaging. The use of rates of atrophy to identify those closer to conversion would inevitably require a period of observation prior to treatment (or randomisation) where serial MR imaging was obtained.

\section{Outcome measures: markers of progression}

Perhaps the greatest interest in using MRI in trials in AD has been as an outcome measure - as a biomarker of progression. A characteristic feature of the disease is the relentless neuronal destruction manifest at a macroscopic level by progressive cerebral atrophy: this atrophy can be tracked using MRI. The safety, availability and relative affordability of MRI makes it feasible for large trials and measurement can be performed at a central site. The key attraction of incorporating MRI measures of progression is to provide a means of identifying diseasemodifying (slowing) effects of a therapy as opposed to temporary symptomatic effects. Although clinical outcomes remain paramount the idea is that imaging biomarkers of progression might provide evidence of disease-slowing earlier or more cost-effectively than clinical outcomes (which potentially require lengthy and complex trials) (6). Biomarkers are often referred to as possible "surrogate" outcome measures for clinical outcomes. In reality imaging biomarkers (or others) are a long way from being used as such but nonetheless they may be useful alongside clinical outcomes rather than replacing them altogether. For biomarkers (imaging or other) to be accepted as surrogate outcomes in AD it is likely multiple results from disease-modifying trials supporting their validity will be required. The two most established markers of progression on MRI are hippocampal and whole brain atrophy rates (6-8). 


\section{Regional measures: hippocampus}

In established $\mathrm{AD}$, mean hippocampal atrophy rates are typically around $4-6 \%$ per year. This contrasts with the rates of only $1-2 \%$ per year in normal subjects (aged around $70-80$ years) $(9,10,11)$. This disease-related difference offers a potential measure which can be used to provide evidence for disease modification. The argument being, that if the treatment were to completely stop pathological progression then atrophy should slow to normal aging rates; with proportional effects on atrophy reflecting different therapeutic effect sizes. The variance in the $\mathrm{AD}$ rates is what determines the ability to detect change (power) and hence the sample sizes needed $(6,12)$. Typically reported standard deviations of AD hippocampal atrophy rates are around 3-3.5\%/yr for a one year study falling to $2-2.5 \% / y r$ with an 18 month study. Hippocampal atrophy rates have been proposed as particularly suitable for early disease where rates of loss are preferentially concentrated in the medial temporal lobe. The entorhinal cortex should theoretically be a more sensitive marker given its early histopathological (tangle) burden (4). However it has been incorporated into fewer trials perhaps because of measurement reliability issues. Most trials have used manual outlining of the hippocampus; more recently semi-automated measures have been used - some of these require landmarks to be placed around the hippocampus from which an algorithm delineates the hippocampal boundaries. Fully automated (template-based) measures have also been developed but have not to date been used in large trials $(3,10)$.

\section{Global brain measures}

Rates of whole brain atrophy have been incorporated into a number of trials in AD. These have the advantage that they incorporate all changes throughout the brain rather than focus on changes in one particular area such as the medial temporal lobe. Equally, however, whole brain atrophy measures incorporate areas that are not particularly affected by the disease, such as the cerebellum or the brain stem. One advantage of measuring the whole brain is the precision of measurement. Whole brain measures, unlike hippocampal measures, have less indistinct and thereby more reliable anatomical boundaries. The use of registration-based measures (e.g the Brain Boundary Shift Integral (BBSI) and Structural Image Evaluation of Normalized Atrophy (SIENA) have allowed whole brain atrophy measures to be automated with a high level of reliability (13). Rates of whole brain atrophy in $\mathrm{AD}$ are typically around $2 \%$ per year, while normal aging rates of atrophy (for a mean age of 70years) are around $0.5 \%$ per year (8). Once again it is this differential between diseaserelated atrophy and normal aging which offers the possibility of providing evidence of a disease-modifying effect of therapy. The standard deviation of the AD brain atrophy rate is typically $\sim 1 \% /$ year for a single centre study of one year or more. These figures mean sample sizes for brain and hippocampal measures are similar $(3,10)$.
Another "global" measure of progression on MRI is the rate of ventricular expansion. This is very non-specific and may be influenced by non-pathological affects, such as hydration. Nonetheless it has the advantage in that it involves a high contrast boundary between CSF and brain and being in the centre of the brain is often less subject to image artefact. Ventricular enlargement in AD is typically around $5 \mathrm{mls}$ per year with normal aging being about $1 \mathrm{ml}$ per year (11).

\section{Automated regional, grey matter and cortical thickness measures}

The development of software that can provide automated or semi-automated measurements of region volumes or cortical thickness, has led to these measures being suggested as outcomes in disease modification trials $(3,4,10)$. Multiple regions may be measured which avoids the limitations of one global or regional measure, nonetheless this raises issues for trial designs that typically pre-specify a single outcome and may require a step-down approach to several measures.

\section{Safety measures}

As we move closer to truly disease modifying therapies, it is likely that these are will have unpredictable safety issues. The use of MRI in the more recent trials has proved to be a sensitive marker of effects that may initially be clinically silent (14). Typically, MRI for safety includes a FLAIR sequence (sensitive to inflammation, infarction and vasogenic oedema) susceptibility-weighted imaging (to detect microhaemorrhages) and some studies incorporate a diffusion sequence. The use of these markers is in their relatively early stages and methods of assessments are still somewhat variable and lack consensus guidelines.

\section{Practical considerations, study design and sample sizes}

The choice of imaging biomarker may depend upon the therapy tested but is often driven by sample size considerations. For trials of 18-months duration 100-200 subjects per arm completing the trial with adequate scans seems to be sufficient to give $90 \%$ power to detect a $20 \%$ slowing of hippocampal or whole brain atrophy - these are much smaller numbers than would be required to show a similar effect size with a clinical outcome $(7,15)$. Head-to head comparisons of different imaging markers are lacking however studies such as the Alzheimer's Disease Neuroimaging Initiative (ADNI) are facilitating direct comparison of the outcome measures discussed above. In addition comparison and correlation with clinical outcomes and with CSF markers, functional imaging and amyloid imaging measures will also be available. Irrespective of outcome measure, the power of the trial depends on the quality of the data which should be ensured by careful qualification of (MRI) sites prior to recruitment and then ongoing quality control and assurance. Patient tolerance is related to the total time in the scanner and with the increased 
use of multiple sequences for safety and efficacy it is important that this time is kept to the minimum, ideally under 30 minutes, to maximise patient compliance and reduce movement artefact. The number and timing of scans and trial duration all have a critical bearing on the power of the study - in the past there was a lack of evidence on which to base these decisions but increasingly it should be possible to make evidence-based decisions on how to use imaging biomarkers most effectively in trials.

\section{Conclusion}

Structural imaging, in particular MRI, has important roles to play in defining the study population in AD and MCI trials. It has a growing role as a safety marker and as an outcome measure. It is likely that multiple different biomarkers will become combined with MRI measures to improve the potential of therapeutic trials to detect disease modification. Interpreting and validating the results of biomarker studies in such trials will be a challenge for the coming years and ultimately will require careful correlation with long term clinical outcomes. Imaging biomarkers may contribute to accelerating the discovery of disease-modifying therapies but it is only once disease-modifying therapies are found that the value of imaging outcomes will really be tested. In the meantime trials are likely to incorporate complementary biomarkers and clinical outcomes as well as MRI measures of atrophy progression.

\section{References}

1. Scheltens P, Fox N, Barkhof F, De Carli C. Structural magnetic resonance imaging in the practical assessment of dementia: beyond exclusion. Lancet Neurol. 2002 May;1(1):13-21.

2. Devanand DP, Pradhaban G, Liu X, Khandji A, De Santi S, Segal S, Rusinek H, Pelton GH, Honig LS, Mayeux R, Stern Y, Tabert MH, de Leon MJ. Hippocampal and entorhinal atrophy in mild cognitive impairment: prediction of Alzheimer disease. Neurology. 2007 Mar 13;68(11):828-36.
3. Desikan RS, Fischl B, Cabral HJ, Kemper TL, Guttmann CR, Blacker D, Hyman BT, Albert MS, Killiany RJ. MRI measures of temporoparietal regions show differential rates of atrophy during prodromal AD. Neurology. 2008 Sep 9;71(11):819-25

4. Du AT, Schuff N, Kramer JH, Ganzer S, Zhu XP, Jagust WJ, Miller BL, Reed BR, Mungas D, Yaffe K, Chui HC, Weiner MW. Higher atrophy rate of entorhinal cortex than hippocampus in AD. Neurology. 2004 Feb 10;62(3):422-7.

5. Ridha BH, Barnes J, Bartlett JW, Godbolt A, Pepple T, Rossor MN, Fox NC Tracking atrophy progression in familial Alzheimer's disease: a serial MRI study. Lancet Neurol. 2006 Oct;5(10):828-34.

6. Fox NC, Cousens S, Scahill R, Harvey RJ, Rossor MN. Using serial registered brain magnetic resonance imaging to measure disease progression in Alzheimer disease: power calculations and estimates of sample size to detect treatment effects. Arch Neurol. 2000 Mar;57(3):339-44.

7. Jack CR Jr, Shiung MM, Gunter JL, O'Brien PC, Weigand SD, Knopman DS, Boeve BF, Ivnik RJ, Smith GE, Cha RH, Tangalos EG, Petersen RC. Comparison of different MRI brain atrophy rate measures with clinical disease progression in AD. Neurology. 2004 Feb 24;62(4):591-600.

8. Fox NC, Black RS, Gilman S, Rossor MN, Griffith SG, Jenkins L, Koller M; AN1792(QS-21)-201 Study. Effects of Abeta immunization (AN1792) on MRI measures of cerebral volume in Alzheimer disease. Neurology. 2005 May 10;64(9):1563-72.

9. Barnes J, Bartlett JW, van de Pol LA, Loy CT, Scahill RI, Frost C, Thompson P, Fox NC. A meta-analysis of hippocampal atrophy rates in Alzheimer's disease. Neurobiol Aging. 2008 Mar 15. [Epub ahead of print]

10. Morra JH, Tu Z, Apostolova LG, Green AE, Avedissian C, Madsen SK, Parikshak N, Toga AW, Jack CR Jr, Schuff N, Weiner MW, Thompson PM; The Alzheimer's Disease Neuroimaging Initiative. Automated mapping of hippocampal atrophy in 1year repeat MRI data from 490 subjects with Alzheimer's disease, mild cognitive impairment, and elderly controls. Neuroimage. 2008 Nov 8. [Epub ahead of print]

11. Schott JM, Price SL, Frost C, Whitwell JL, Rossor MN, Fox NC. Measuring atrophy in Alzheimer disease: a serial MRI study over 6 and 12 months. Neurology. $2005 \mathrm{Jul}$ 12;65(1):119-24.

12. Schott JM, Frost C, Whitwell JL, Macmanus DG, Boyes RG, Rossor MN, Fox NC.Combining short interval MRI in Alzheimer's disease: Implications for therapeutic trials. J Neurol. 2006 Sep;253(9):1147-53.

13. Smith SM, Rao A, De Stefano N, Jenkinson M, Schott JM, Matthews PM, Fox NC. Longitudinal and cross-sectional analysis of atrophy in Alzheimer's disease: crossvalidation of BSI, SIENA and SIENAX. Neuroimage. 2007 Jul 15;36(4):1200-6. Epub 2007 Apr 27

14. Grundman M and Black R. Clinical trials of Bapineuzumab, a beta-amyloid-targeted immunotherapy in patients with mild to moderate Alzheimer's disease. Alzheimer's and Dementia. 2008 July; 4(4) Suppl 2:T166

15. Ridha BH, Anderson VM, Barnes J, Boyes RG, Price SL, Rossor MN, Whitwell JL, Jenkins L, Black RS, Grundman M, Fox NC. Volumetric MRI and cognitive measures in Alzheimer disease: comparison of markers of progression. J Neurol. 2008 Apr;255(4):567-74 